B-cell lymphomas. N. Engl. J. Med. 359:2313-2323.

11. Byrd, J.C., et al. 2006. Select high-risk genetic features predict earlier progression following chemoimmunotherapy with fludarabine and rituximab in chronic lymphocytic leukemia: justification for risk-adapted therapy. J. Clin. Oncol. 24:437-443.

12. Johnson, P., and Glennie, M. 2003. The mechanisms of action of rituximab in the elimination of tumor cells. Semin. Oncol. 30:3-8.

13. Chan, H.T., et al. 2003. CD20-induced lymphoma cell death is independent of both caspases and its redistribution into triton $\mathrm{X}-100$ insoluble membrane rafts. Cancer Res. 63:5480-5489.

14. Ivanov, A., Krysov, S., Cragg, M.S., and Illidge, T. 2008. Radiation therapy with tositumomab (B1) antiCD20 monoclonal antibody initiates extracellular signal-regulated kinase/mitogen-activated protein kinase-dependent cell death that overcomes resis- tance to apoptosis. Clin. Cancer Res. 14:4925-4934.

15. Cragg, M.S., and Glennie, M.J. 2004. Antibody specificity controls in vivo effector mechanisms of anti-CD20 reagents. Blood. 103:2738-2743.

16. Beers, S.A., et al. 2008. Type II (tositumomab) antiCD20 monoclonal antibody out performs type I (rituximab-like) reagents in B-cell depletion regardless of complement activation. Blood. 112:4170-4177.

17. Ivanov, A., et al. 2009. Monoclonal antibodies directed to CD20 and HLA-DR can elicit homotypic adhesion followed by lysosome-mediated cell death in human lymphoma and leukemia cells. J. Clin. Invest. 119:2143-2159.

18. Cerisano, V., et al. 2004. Molecular mechanisms of CD99-induced caspase-independent cell death and cell-cell adhesion in Ewing's sarcoma cells: actin and zyxin as key intracellular mediators. Oncogene. 23:5664-5674

19. Fehrenbacher, N., et al. 2008. Sensitization to the lysosomal cell death pathway by oncogene-induced down-regulation of lysosome-associated membrane proteins 1 and 2. Cancer Res. 68:6623-6633.

20. Davis, T.A., et al. 2004. The radioisotope contributes significantly to the activity of radioimmunotherapy. Clin. Cancer Res. 10:7792-7798.

21. Horning, S.J., et al. 2005. Efficacy and safety of tositumomab and iodine-131 tositumomab (Bexxar) in B-cell lymphoma, progressive after rituximab. J. Clin. Oncol. 23:712-719.

22. Ostenfeld, M.S., et al. 2008. Anti-cancer agent siramesine is a lysosomotropic detergent that induces cytoprotective autophagosome accumulation. Autophagy. 4:487-499.

23. Groth-Pedersen, L., Ostenfeld, M., Høyer-Hansen, M., Nylandsted, J., and Jäättelä, M. 2007. Vincristine induces dramatic lysosomal changes and sensitizes cancer cells to lysosome destabilizing siramesine. Cancer Res. 67:2217-2225.

\title{
EBAG9 tempers lymphocyte killing activity
}

Gaël Ménasché ${ }^{1,2}$ and Geneviève de Saint Basile ${ }^{1,2}$

1INSERM U768, Paris, France. ²Faculté de Médecine, Université Paris Descartes, Paris, France.

\begin{abstract}
The cytotoxic activity of lymphocytes is crucial for immune surveillance and homeostasis. Several independent, naturally occurring genetic models characterized by defects in granule trafficking or exocytosis have helped to decipher the multiple steps and molecules that regulate the cytotoxic process. The study by Rüder and colleagues in this issue of the JCI shows that an engineered absence of EBAG9, previously reported as a tumor-associated antigen, enhances cytotoxic activity of CTLs but not NK cells, likely acting on the endosomal-lysosomal trafficking of the cytotoxic effectors (see the related article beginning on page 2184). This finding adds a new piece to the puzzle of complex mechanisms that tightly regulate the capacity of the cytotoxic response and suggests a new target to negatively modulate CTL responsiveness.
\end{abstract}

CTLs and NK cells (collectively known as "cytotoxic lymphocytes") are major players in the body's defense against viral infection and cancer via their ability to seek out and kill infected or tumorigenic cells. Although CTLs are activated by specific antigen recognition, the cytotoxic activity of NK cells is initiated by specific activating receptors or combinations thereof and is inhibited by self MHC class I recognition. Once cyto-

Conflict of interest: The authors have declared that no conflict of interest exists.

Nonstandard abbreviations used: AP1, adaptor protein 1 ; BLOC-1, biogenesis of lysosome-related organelles complex 1; EBAG9, estrogen receptor-binding fragment-associated antigen 9; ESCRT, endosomal sorting complex required for transport; HLH, hemophagocytic lymphohistiocytosis; HPS2, HermanskyPudlak syndrome type 2; IS, immunological synapse; LAMP, lysosomal-associated membrane protein; LCMV, lymphocytic choriomeningitis virus; LRO, lysosomerelated organelle; LYST, lysosomal trafficking regulator. Citation for this article: J. Clin. Invest. 119:2136-2140 (2009). doi:10.1172/JCI40270. toxic cells have recognized their targets and formed a conjugate, the trafficking of granule components, including perforin and granzymes, to the immunological synapse (IS) between the cell and its target, leads to the delivery of perforin and granzymes into the target cells and subsequent target destruction through apoptosis $(1,2)$.

Studies of natural or engineered mutants involving cytotoxic function tell us a great deal about the in vivo function of this pathway. So far, only genetic diseases leading to the loss of cytotoxic function have been reported (3). These studies have revealed the tremendous importance of this cytotoxic pathway in immune homeostasis. Congenital defects that lead to either impaired perforin function or its dysregulated release result in the specific severe condition hemophagocytic lymphohistiocytosis (HLH) syndrome. This syndrome stems from the inability of activated cytotoxic cells to clear antigen- presenting targets. The failure to clear antigen causes unremitting polyclonal $\mathrm{CD}^{+} \mathrm{T}$ cell expansion, activation, and infiltration of visceral organs associated with macrophage activation, with the deleterious release of multiple inflammatory cytokines including IFN- $\gamma(3,4)$.

In addition, the studies of these natural mutants both in humans and in mice have contributed to the characterization of critical effectors of the cytotoxic machinery and their function. They also provided evidence of the exquisite regulation of this process, which occurs in successive steps following cell activation. For instance, studies of Griscelli syndrome type 2, a rare condition characterized by partial albinism and occurrence of an HLH syndrome (5), and familial HLH type 3 (FHL3) (6) have shown the critical role of two proteins, the small GTPase Rab27a and the priming factor Munc13-4, at a late step of the secretory pathway. Rab27a regulates the tethering of mature cytotoxic granules that have polarized at the IS, whereas Munc13-4 primes the docked cytotoxic granules before their fusion with the plasma membrane at the IS. Other proteins, for which their defect impairs lymphocyte cytotoxic activity, likely regulate an upstream step in the cytotoxic pathway. They include adapter protein 3 (AP3), which is deficient in Hermansky-Pudlak syndrome type 2 (HPS2) $(7,8)$, and the lysosomal trafficking regulator (LYST) protein, which is deficient in Chediak-Higashi syndrome $(9,10)$. Their 


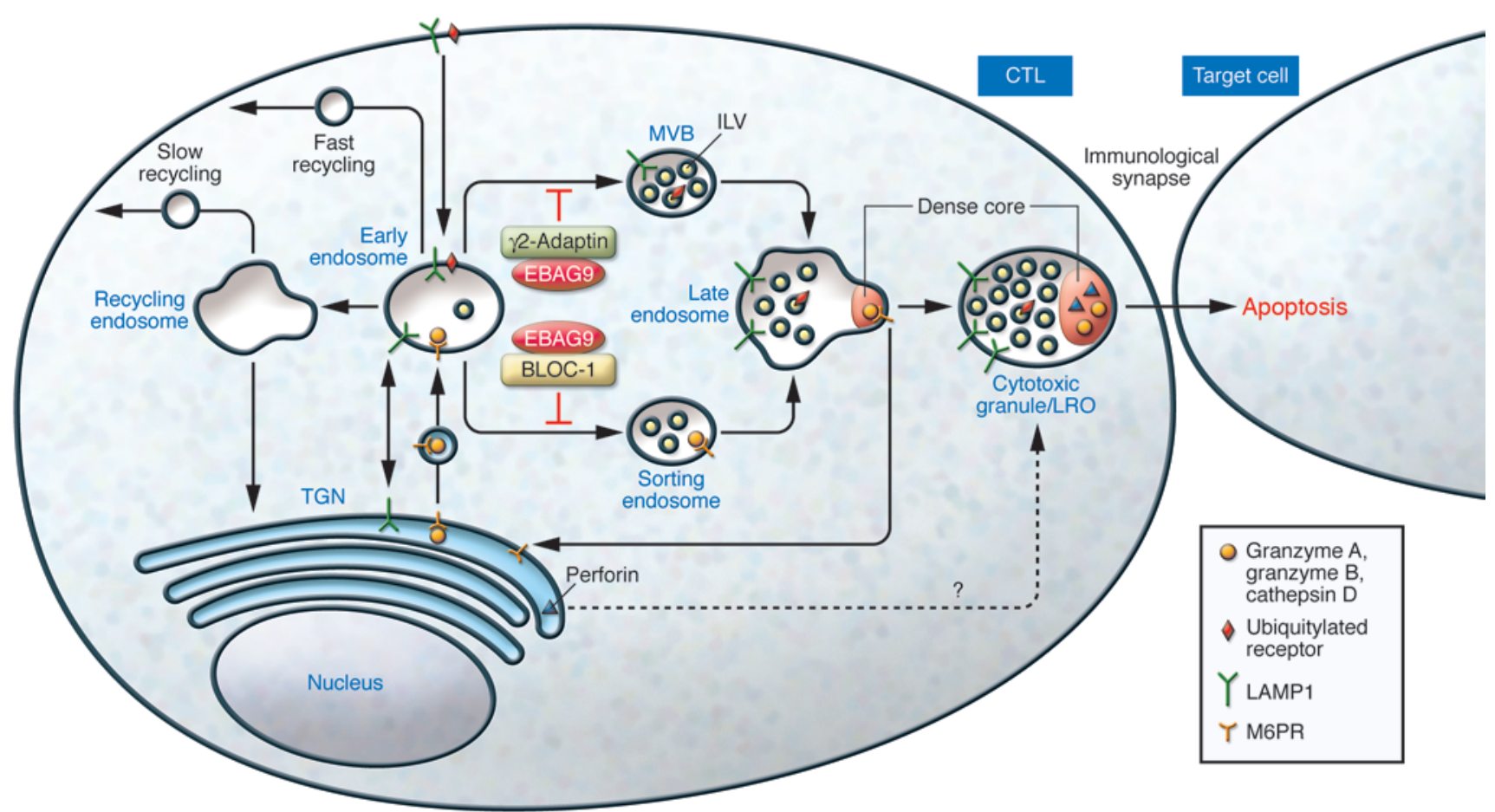

\section{Figure 1}

Sorting proteins to the cytotoxic granules/secretory lysosomes. The newly synthesized soluble proteins granzymes A and B acquire the mannose-6-phosphate signal in the Golgi complex, enabling their association with the mannose-6-phosphate receptor (M6PR) in the trans-Golgi network (TGN) and transport to early endosomes before they are sorted to late endosomes. LAMP1 is sorted by AP complexes (not shown) and transported from the plasma membrane to the endosomes, preferentially to the perimeter membrane of the multivesicular bodies (MVBs) in the sorting endosome and then to the late endosome and cytotoxic granule/LRO. Early endosomes sort cargoes to different destinations. Cargoes can be recycled back to the plasma membrane via fast or slow pathways, delivered to the lysosomes through sorting endosomes and multivesicular bodies, or sorted to the trans-Golgi network. BLOC-1 is involved in the exit of cargoes from an early endosome subdomain toward intermediate/sorting endosomes. $\gamma 2$-Adaptin has been reported (19) to associate with the ubiquitin machinery, which directs cargo incorporation into intraluminal vesicles (ILVs) of multivesicular bodies by the ESCRT machinery. The study by Rüder et al. in this issue of the $\mathrm{JCl}(11)$ reports that EBAG9 associates with and inhibits $\gamma 2$-adaptin and associates with two BLOC-1 subunits. Loss of EBAG9 enhances the cytotoxic activity of T lymphocytes, likely acting on the endosomal-lysosomal trafficking of the cytotoxic effectors. Although the detailed mechanism accounting for the transport of perforin from the trans-Golgi network to late endosomes is still poorly understood, the findings of Rüder et al. (11) suggest a connection among granzymes and perforin trafficking, the BLOC-1 components, and the ESCRT machinery.

respective defects include missorting of transmembrane proteins to lysosomes and an increase in the size of cytotoxic granules. Although the precise functions of these effectors in the cytotoxic pathway remain poorly understood, both proteins likely regulate lysosomal biogenesis and membrane fusion along the cytotoxic pathway.

The proteins mentioned above are all required for proper cytotoxic activity of lymphocytes. In contrast, Rüder and colleagues report in this issue of the JCI that estrogen receptor-binding fragment-associated antigen 9 (EBAG9) acts as a negative regulator of cytotoxic function (11). Through the generation of Ebag9-deficient mice, they show that loss of EBAG9 enhances the release of cytotoxic granules in vitro as well as the cytotoxic capacity of CTLs from deficient mice in vivo. The increase in the sorting of cytotoxic effector molecules to cytotoxic granules associated with EBAG9 deficiency, together with the demonstration that EBAG9 interacts with the $\gamma 2$-adaptin adapter protein, indicate that EBAG9 plays a pivotal role in the sorting of endosomal-lysosomal proteins. Therefore, the work presented by Rüder and colleagues introduces a new player involved in the tuning of the cytotoxic function of lymphocytes. However, it remains to be determined what the precise molecular mechanism is that underlies the observed phenotype; there is no evidence of immunopathogenesis in EBAG9-deficient mice. In the future, it may be worth studying the consequences of lymphocytic choriomeningitis virus (LCMV) infection in EBAG9-deficient mice, as in this setting mice impaired in cytotoxic lympho- cyte function demonstrate a clear immune disease, i.e., HLH (12). An "opposite" phenotype, with limitation of the immune response to pathogen, might be seen, as we discuss below. The trafficking of lysosomal proteins in other cells, such as mast cells, may also be increased in EBAG9-deficient mice, potentially exacerbating an anaphylactic reaction.

\section{Biogenesis of cytotoxic granules}

Mature cytotoxic granules are lysosomerelated organelles (LROs) that act as dualfunction organelles, carrying out both degradative and secretory functions (2, 13). Cytotoxic granules exhibit an electron-dense core that contains most of the cytotoxic proteins, including perforin and granzymes, similar to secretory organelles. The core is surrounded by a multivesicu- 
lar/lamellar region typical of lysosomes. The cytotoxic granules have a low lumenal $\mathrm{pH}$ and contain lysosomal acid hydrolases such as cathepsin D and receptors such as a member of the lysosomal-associated membrane protein (LAMP) family. The biogenesis of these cytotoxic organelles is not well characterized, but, as in the case of other LROs, they likely use the cooperation of ubiquitous trafficking machineries with cell type-specific cargoes (14) (Figure 1). Granzymes, similar to other soluble lysosomal proteins, are modified during biosynthesis by the addition of a mannose6-phosphate moiety, which is recognized by mannose-6-phosphate receptors. These transmembrane receptors cycle among the trans-Golgi network and early and late endosomes, carrying soluble proteins to the lysosome structures. However, the pathway used by perforin to reach the lysosomes is still unknown (Figure 1). LAMPs do not require sorting receptors, since they have a tyrosine-based motif that directly interacts with multimeric cytosolic AP1, AP2, and AP3. Biogenesis of LROs in different cell types also depends on the function of the biogenesis of lysosome-related organelles complex-1 (BLOC-1), -2 , and -3 protein complexes (15). These protein complexes have been largely defined from the studies of the various genes leading to the phenotype of HPS or a similar disorder in mice (14). Of note, the AP3 complex was shown to interact with BLOC-1 on early endosomes to facilitate the trafficking of LAMP to the lysosome (16). In addition, cytotoxic cells, similar to all cell types, may use the endosomal sorting complex required for transport (ESCRT) (17). Ubiquitylation targets proteins to this pathway. ESCRT complexes recognize ubiquitylated proteins on early endosomes, which are subsequently invaginated into newly forming multivesicular bodies. Ubiquitylated proteins accumulate in these intralumenal vesicles of endosomes and then are sorted to late endosomes and lysosomes. So far, none of the CTL-restricted lysosomal proteins have been found to follow this ESCRT sorting pathway to be incorporated into the vesicular structure of the granules.

Thus different machineries may participate along the endosomal pathway in the sorting of cargoes involved in LRO biogenesis. The new data reported by Rüder et al. (11) provide some evidence of a link among EBAG9, the BLOC-1 components, the ESCRT machinery, and the biogenesis of cytotoxic granules (Figure 1).

\section{Which step of the cytotoxic pathway is regulated by EBAG9?}

Rüder and colleagues used various approaches to determine which step of cytotoxic granule biogenesis is regulated by EBAG9 expression (11). Their findings provide compelling evidence that EBAG9 does not directly regulate the secretion of secretory lysosomes, but rather acts upstream of the exocytic process, in the trafficking of the cytotoxic components along the endosomal-lysosomal pathway. They observed that the loss of EBAG9 enhanced the sorting of granzyme $B$ and of the acid hydrolase cathepsin D to the secretory lysosomes. In contrast, the sorting of lysosomal membrane marker LAMP1, which uses an AP-dependent pathway, was reduced in EBAG9-deficient cells. Interestingly, they report (although this data is not shown) that the sorting of perforin was also enhanced, suggesting that the sorting of perforin may occur via the same trafficking pathway as the sorting of granzymes.

A role for EBAG9 in the trafficking between endosomal and lysosomal compartments is further supported by the finding that EBAG9 interacts with different proteins involved in the sorting of proteins at an early step along this route (11). By yeast two-hybrid analysis, Rüder et al. identified EBAG9 as interacting with two subunits of BLOC-1, snapin and BLOS2, although the authors failed to confirm this interaction by coimmunoprecipitation of the proteins expressed at the endogenous level (11). BLOC-1 mainly localizes to a subdomain of early endosomes and is involved in the exit of selective cargoes from this domain to maturing LROs (15). Although loss of BLOC- 1 components has never been shown before to be required for cytotoxic function (18), the possibility that EBAG9 regulates a BLOC-1dependent cytotoxic granule maturation, as suggested by these data, remains an attractive hypothesis. Another role of snapin in membrane fusion, through its interaction with the SNARE proteins (SNAP-25 and -23), has also been reported (15). This interaction may also participate in cargo exit or travel by regulating membrane fusion events.

In addition, Rüder et al. show that EBAG9 not only associates with BLOC-1 components but also interacts with the adapter protein $\gamma 2$-adaptin (11). $\gamma 2$-Adaptin is a unit of the AP complexes, but unlike the other members of the adapter protein family, $\gamma 2$-adaptin has a ubiquitin-binding abil- ity and can recruit the ubiquitination machinery (19). Thus, by interacting with $\gamma 2$-adaptin, EBAG9 may participate in the endosomal/multivesicular body sorting and trafficking system within the ESCRT machinery. This function again localizes EBAG9 at the exit of cargo from the early endosome. Of note, depletion of $\gamma 2$-adaptin results in an enlargement of endosomal vesicles and the accumulation in these vesicles of proteins destined to lysosomal degradation (19). In contrast loss of EBAG9 enhances proteolytic processing of the lysosomal protein cathepsin D and decreases cytotoxic granule size. This opposite phenotype supports a role of EBAG9 as a negative regulator of $\gamma 2$-adaptin.

Although our present knowledge of the molecular mechanisms that finely regulate the intracellular trafficking of proteins in CTLs precludes further assumption on the precise role of EBAG9 in these cells, some points should be underscored. First, the interaction of EBAG9 with two BLOC-1 components and with $\gamma 2$-adaptin, if functionally relevant, indicate that EBAG9 regulates sorting of proteins from early endosomes, potentially through two different sorting pathways. Second, it is intriguing to note that the association of an increase in cytotoxic component trafficking, an enhanced cytotoxic function, and a reduced size of cytotoxic granules, which all result from loss of function of EBAG9, inversely mirror the phenotypes evoked by functional loss of LYST in Chediak-Higashi syndrome and of AP3 in HPS2 $(8,20,21)$. Both of these proteins are known to participate in the sorting of lysosomal proteins $(9,15)$. LYST was also recently reported to interact with HRS and LIP5, two ESCRTassociated proteins, and aberrant enlarged vacuolar structures are found when HRS is deficient $(17,22)$. These observations suggest opposite functions of EBAG9 (negative) and LYST and AP3 (positive) in the regulatory sorting of endosomal proteins into lysosomes.

Together with previous observations that AP3 is able to interact with BLOC-1 and that both HRS and snapin were shown to interact with SNAP-25 in neuroendocrine cells $(15,22)$, the work of Rüder et al. (11) further suggests that the formation of LROs, among them cytotoxic granules, is more complicated than that of modified lysosomes. Their work further suggests that these granules mature by the addition of cargoes sorted through different pathways and that molecular connections 
between distinct sorting machineries may potentially exist.

Together with the previous work of Rüder and colleagues, which shows that overexpression of EBAG9 in neuroendocrine cells leads to a decrease in high $\mathrm{K}^{+}$-induced granule release (23), a role for EBAG9 in the negative regulation of the cytotoxic activity of lymphocytes is therefore most likely.

\section{Does EBAG9 deficiency affect serial killing capacity of CTLs?}

CTLs are able to successively kill several target cells in a short time period. The iterative killing ability of these cells depends on the combination of two factors: following target cell recognition, TCR triggering leads to the release of only a fraction of the mature cytotoxic granules from CTLs, and at the same time cytotoxic proteins are synthesized to refill the store of cytotoxic components. Regulation of the quantum of cytotoxic granules secreted at the IS may occur at a very late stage of cytotoxic granule maturation, when these granules are polarized together with endosomal exocytic vesicles at the IS and fuse with these vesicles prior to secretion (24). Thus, a pool of cytotoxic granules ready to release their contents persists in CTLs following killing of a first target. This pool can then be used to successively kill other encountered targets. An interesting question is whether the rate of protein synthesis is sufficient to compensate for the increase in trafficking and therefore release of cytolytic components when EBAG9 is defective.

\section{Could excessive cytotoxic function of lymphocytes disturb immune homeostasis?}

The granule-dependent cytotoxic activity of lymphocytes plays a major role in the elimination of infected cells, simultaneously regulating the magnitude of the immune response. LCMV infection in mice deficient for the cytotoxic activity of lymphocytes reproduces the immunopathology of HLH observed in humans $(12,25)$. Not all strains of viruses can trigger such immune manifestations in mice deficient for cytotoxic lymphocyte activity. The fact that clearance of LCMV infection is absolutely dependent on perforin-mediated cytolysis may be an important parameter. Thus, in the context of LCMV infection, the balance between cytotoxicity, antigen presentation, and cytokine production determines the outcome of the immune response.
Rüder and colleagues report that EBAG9deficient mice exhibited augmented CTL clearance of antigen when immunized with Listeria monocytogenes (11). It may be worth studying this mouse model to determine whether, during LCMV infection, enhanced viral antigen clearance has any consequences on the magnitude or the quality of the immune response. One may expect that, in this setting, a rapid elimination of antigens would diminish the expansion of antigen-specific effector cells or the associated Th1 cytokine production (e.g., IFN- $\gamma$, TNF- $\alpha$, IL-6) and also ultimately dampen the generation of memory $\mathrm{CD}^{+} \mathrm{T}$ cells and modify the immunodominance hierarchy of the $\mathrm{T}$ cell response (i.e., the specificity of the epitopes present in a given antigen that will preferentially elicit the $\mathrm{CD}^{+} \mathrm{T}$ cell response), as previously shown in mice deficient for IFN- $\gamma$ (26). Alternatively, the increase in the cytotoxic granule release by CTLs may cause toxic bystander effects, leading to destruction of neighboring cells or tissue infiltrated by the effector cytotoxic cells.

To our knowledge, this report (11) describes the second non-receptor protein whose silencing potentiates $\mathrm{T}$ lymphocyte cytotoxicity. Janus kinase and microtubule-interacting protein 1 was previously described as able to restrain $\mathrm{T}$ cell-mediated cytotoxicity, potentially acting on cytotoxic granule transport along microtubules (27). Thus, EBAG9 appears to be a new player able to downregulate the process of lymphocyte cytotoxic activity. It suggests that CTL activity, as other major effector pathways of the immune response, is controlled at multiple checkpoints. It is not foolish to predict a future description of several of these checkpoints.

\section{Acknowledgments}

We thank Alain Fischer for critical reading of this manuscript. The authors are supported by grants from the French National Institute for Health and Medical Research (INSERM), the French National Research Agency (ANR), and the Fondation pour la Recherche Médicale (FRM).

Address correspondence to: Geneviève de Saint Basile, INSERM U768, 149 rue de Sèvres, 75015, Paris, France. Phone: 33-144-49-50-80; Fax: 33-1-42-73-06-40; E-mail: genevieve.de-saint-basile@inserm.fr.

1. Voskoboinik, I., Smyth, M.J., and Trapani, J.A. 2006. Perforin-mediated target-cell death and immune homeostasis. Nat. Rev. Immunol. 6:940-952.

2. Stinchcombe, J.C., and Griffiths, G.M. 2007. Secretory mechanisms in cell-mediated cytotoxicity. Annu. Rev. Cell Dev. Biol. 23:495-517.

3. Menasche, G., Feldmann, J., Fischer, A., and de Saint Basile, G. 2005. Primary hemophagocytic syndromes point to a direct link between lymphocyte cytotoxicity and homeostasis. Immunol. Rev. 203:165-179.

4. Fischer, A., Latour, S., and de Saint Basile, G. 2007. Genetic defects affecting lymphocyte cytotoxicity. Curr. Opin. Immunol. 19:348-353.

5. Ménasché, G., et al. 2000. Mutations in RAB27A cause Griscelli syndrome associated with hemophagocytic syndrome. Nat. Genet. 25:173-176.

6. Feldmann, J., et al. 2003. Munc13-4 is essential for cytolytic granules fusion and is mutated in a form of familial hemophagocytic lymphohistiocytosis (FHL3). Cell. 115:461-473.

7. Dell'Angelica, E.C., Shotelersuk, V., Aguilar, R.C., Gahl, W.A., and Bonifacino, J.S. 1999. Altered trafficking of lysosomal proteins in HermanskyPudlak syndrome due to mutations in the beta $3 \mathrm{~A}$ subunit of the AP-3 adaptor. Mol. Cell. 3:11-21.

8. Clark, R.H., et al. 2003. Adaptor protein 3-dependent microtubule-mediated movement of lytic granules to the immunological synapse. Nat. Immunol. 4:1111-1120.

9. Faigle, W., et al. 1998. Deficient peptide loading and MHC class II endosomal sorting in a human genetic immunodeficiency disease: the chediakhigashi syndrome. J. Cell Biol. 141:1121-1134.

10. Ward, D.M., Griffiths, G.M., Stinchcombe, J.C., and Kaplan, J. 2000. Analysis of the lysosomal storage disease Chediak-Higashi syndrome. Traffic. 1:816-822.

11. Rüder, C., et al. 2009. The tumor-associated antigen EBAG9 negatively regulates the cytolytic capacity of mouse CD8 ${ }^{+} \mathrm{T}$ cells. J. Clin. Invest. 119:2184-2203.

12. Jordan, M.B., Hildeman, D., Kappler, J., and Marrack, P. 2004. An animal model of hemophagocytic lymphohistiocytosis (HLH): CD8+ T cells and interferon gamma are essential for the disorder. Blood. 104:735-743.

13. Bossi, G., and Griffiths, G.M. 2005. CTL secretory lysosomes: biogenesis and secretion of a harmful organelle. Semin. Immunol. 17:87-94.

14. Raposo, G., Marks, M.S., and Cutler, D.F. 2007. Lysosome-related organelles: driving post-Golgi compartments into specialisation. Curr. Opin. Cell Biol. 19:394-401.

15. Setty, S.R., et al. 2007. BLOC-1 is required for cargospecific sorting from vacuolar early endosomes toward lysosome-related organelles. Mol. Biol. Cell. 18:768-780.

16. Di Pietro, S.M., et al. 2006. BLOC-1 interacts with BLOC-2 and the AP- 3 complex to facilitate protein trafficking on endosomes. Mol. Biol. Cell. 17:4027-4038.

17. Williams, R.L., and Urbe, S. 2007. The emerging shape of the ESCRT machinery. Nat. Rev. Mol. Cell Biol. 8:355-368.

18. Bossi, G., et al. 2005. Normal lytic granule secretion by cytotoxic $\mathrm{T}$ lymphocytes deficient in BLOC- $1,-2$ and -3 and myosins Va, VIIa and XV. Traffic. 6:243-251.

19. Rost, M., Doring, T., and Prange, R. 2008. gamma2Adaptin, a ubiquitin-interacting adaptor, is a substrate to coupled ubiquitination by the ubiquitin ligase Nedd 4 and functions in the endosomal pathway. J. Biol. Chem. 283:32119-32130.

20. Barbosa, M.D.F.S., et al. 1996. Identification of the homologous beige and Chediak-Higashi syndrome genes. Nature. 382:262-265.

21. Nagle, D.L., et al. 1996. Identification and mutation analysis of the complete gene for ChediakHigashi syndrome. Nat. Genet. 14:307-311. 
22. Tchernev, V.T., et al. 2002. The Chediak-Higashi protein interacts with SNARE complex and signal transduction proteins. Mol. Med. 8:56-64.

23. Ruder, C., et al. 2005. EBAG9 adds a new layer of control on large dense-core vesicle exocytosis via interaction with Snapin. Mol. Biol. Cell. 16:1245-1257.

24. Menager, M.M., et al. 2007. Secretory cytotoxic gran- ule maturation and exocytosis require the effector protein hMunc13-4. Nat. Immunol. 8:257-267.

25. Pachlopnik Schmid, J., et al. 2008. A Griscelli syndrome type 2 murine model of hemophagocytic lymphohistiocytosis (HLH). Eur. J. Immunol. 38:3219-3225

26. Badovinac, V.P., Tvinnereim, A.R., and Harty, J.T.
2000. Regulation of antigen-specific CD8+ T cell homeostasis by perforin and interferon-gamma. Science. 290:1354-1358.

27. Libri, V., et al. 2008. Jakmip1 is expressed upon $\mathrm{T}$ cell differentiation and has an inhibitory function in cytotoxic T lymphocytes. J. Immunol. 181:5847-5856.

\title{
Haptoglobin halts hemoglobin's havoc
}

\author{
Gregory J. Kato \\ Sickle Cell Vascular Disease Section, Pulmonary and Vascular Medicine Branch, National Heart, Lung, and Blood Institute, and \\ Critical Care Medicine Department, Clinical Center, National Institutes of Health, Bethesda, Maryland, USA.
}

\begin{abstract}
Hemoglobin $(\mathrm{Hb})$ is crucial to the function of the red blood cell. However, when it is released during intravascular hemolysis from the cell into blood plasma, it produces a state of NO depletion, oxidant stress, and vascular dysfunction, including hypertension. In their study reported in this issue of the JCI, Boretti and colleagues used canine and guinea pig models to demonstrate that pharmacological doses of glucocorticoid can increase the plasma levels of haptoglobin $(\mathrm{Hp})$, the principal plasma-binding protein for free $\mathrm{Hb}$ (see the related article beginning on page 2271). Hp prevented $\mathrm{Hb}$-induced hypertension and the generation of oxidant damage to the kidney. Neutralization of free $\mathrm{Hb}$ appears to be part of the downstream antiinflammatory properties of glucocorticoid.
\end{abstract}

Hemoglobin $(\mathrm{Hb})$ plays a critical role in vascular function - it carries oxygen and mediates adaptive vasodilation via $\mathrm{NO}$ signaling, most likely via nitrite reductase activity, under hypoxic conditions (1). When red blood cells lyse during intravascular hemolysis, $\mathrm{Hb}$ crosses the red blood cell membrane into plasma. Once decompartmentalized from red blood cells, free $\mathrm{Hb}$ potently induces oxidative stress and scavenges $\mathrm{NO}$, reducing the bioavailability of this critical antioxidant and master regulator of vascular homeostasis, wreaking havoc on vascular health (2). In this issue of the JCI, Boretti et al. show, in canine and guinea pig models, that pharmacological activation of the glucocorticoid pathway induces expression of the $\mathrm{Hb}$-scavenging protein haptoglobin $(\mathrm{Hp})$, which sequesters cellfree plasma $\mathrm{Hb}$ and neutralizes much of its vasculotoxic oxidative stress, protecting against systemic hypertension and other vasculopathic outcomes (3).

Conflict of interest: The author has received research support in the form of a Collaborative Research and Development Agreement between the National Institutes of Health and Ikaria-INO Therapeutics.

Nonstandard abbreviations used: $\mathrm{Hb}$, hemoglobin; $\mathrm{Hp}$, haptoglobin.

Citation for this article: J. Clin. Invest. 119:2140-2142 (2009). doi:10.1172/JCI40258.
Hemolysis, NO scavenging, oxidative stress, and clinical outcomes

For decades, it has been known that $\mathrm{Hb}$ is a highly efficient scavenger of NO in vitro. However, only in recent years has it become more widely appreciated that cellfree $\mathrm{Hb}$ scavenges $\mathrm{NO}$ in vivo in human disease and produces clinical manifestations (Figure 1) (2). The depletion of NO, a crucial endogenous antioxidant, is compounded by the oxidative stress induced by decomposition of $\mathrm{Hb}$ into heme and elemental iron, highly oxidative co-conspirators against vascular health. Indeed, not only does a canine model of intravascular hemolysis acutely demonstrate the induction of systemic and pulmonary hypertension but also impaired creatinine clearance, presumably due to decreased renal blood flow (4). Overlapping syndromes have been observed in other human examples of acute hemoglobinemia (excess $\mathrm{Hb}$ in the blood plasma), such as the acute hemolytic transfusion reaction (abrupt hemolysis of transfused red blood cell), or following infusion of the first generation of cell-free blood substitutes (2). The acute experimental canine vasculopathy is partially reversed by administration of $\mathrm{NO}$ (4) or alternatively, as described in this issue by Boretti et al., by glucocorticoid-induced production of endogenous $\mathrm{Hp}$ (3).
Sickle-cell disease has provided the most well-documented example of chronic hemolysis and clinical vasculopathy, with markers of hemolysis-induced NO scavenging statistically linked with pulmonary hypertension, leg ulceration, priapism, and cerebrovascular disease (5). Reports also have begun to accumulate for these same exact complications, arising in other non-sickling, acute, and chronic hemolytic disorders, supporting a contributory role for hemolysis in the pathophysiology of this vasculopathy syndrome (5). This growing list includes thalassemia, autoimmune hemolytic anemia, malaria, paroxysmal nocturnal hemoglobinuria, unstable hemoglobinopathy, and hereditary membranopathies (5).

The biological importance of these pathways is emphasized by the redundant and overlapping mechanisms that have evolved to detoxify cell-free plasma $\mathrm{Hb}$. First among these is Hp, a tetrameric plasma glycoprotein that binds cell-free plasma $\mathrm{Hb}$ and quickly escorts it to the CD163 protein, in which the Hp-Hb complex is avidly bound and cleared from plasma, depleting plasma Hp in the process (6) (Figure 1). Boretti et al. (3) found that $\mathrm{Hp}$ binding to $\mathrm{Hb}$ is sufficient to prevent the generation of oxidant species from cell-free $\mathrm{Hb}$ that would otherwise mediate hypertension and other adverse vascular outcomes, perhaps in part by sequestering $\mathrm{Hb}$ in a high-molecularweight complex that would not extravasate into the subendothelial space. Interestingly, Boretti et al. also show that Hp-bound $\mathrm{Hb}$ has a very high oxygen affinity, which should correspond to increased nitrite reductase activity (1), potentially stimulating the activity of $\mathrm{Hp}-\mathrm{Hb}$ complexes to produce the endogenous antioxidant $\mathrm{NO}$ from nitrite. As part of the teleological evidence of the biological influence of cell-free plasma $\mathrm{Hb}$, additional $\mathrm{Hb}$-binding pathways 